\title{
Development and validation of a novel survival model for acute myeloid leukemia based on autophagy-related genes
}

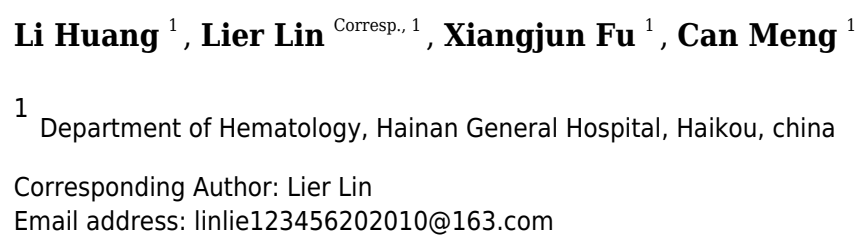

Background: Acute myeloid leukemia (AML) is one of the most common blood cancers, and is characterized by impaired hematopoietic function and bone marrow (BM) failure. Under normal circumstances, autophagy may suppress tumorigenesis, however under the stressful conditions of late stage tumor growth autophagy actually protects tumor cells, so inhibiting autophagy in these cases also inhibits tumor growth and promotes tumor cell death. Methods: AML gene expression profile data and corresponding clinical data were obtained from the Cancer Genome Atlas (TCGA) and Gene Expression Omnibus (GEO) databases, from which prognostic-related genes were screened to construct a risk score model through LASSO and univariate and multivariate Cox analyses. Then the model was verified in the TCGA cohort and GEO cohorts. In addition, we also analyzed the relationship between autophagy genes and immune infiltrating cells and therapeutic drugs. Results: We built a model containing 10 autophagy-related genes to predict the survival of AML patients by dividing them into high- or low-risk subgroups. The high-risk subgroup was prone to a poorer prognosis in both the training TCGA-LAML cohort and the validation GSE37642 cohort. Univariate and multivariate Cox analysis revealed that the risk score of the autophagy model can be used as an independent prognostic factor. The high-risk subgroup had not only higher fractions of CD4 naïve T cell, NK cell activated, and resting mast cells but also higher expression of immune checkpoint genes CTLA4 and CD274. Last, we screened drug sensitivity between high- and low-risk subgroups. Conclusion: The risk score model based on 10 autophagy-related genes can serve as an effective prognostic predictor for AML patients and may guide for patient stratification for immunotherapies and drugs. 
1 Development and validation of a novel survival model for Acute myeloid

\section{2 leukemia based on autophagy-related genes}

3

4 Li Huang ${ }^{1}$, Lier Lin ${ }^{1 *}$, Xiangjun Fu ${ }^{1}$, Can Meng ${ }^{1}$

51 Department of Hematology, Hainan General Hospital, Haikou city, China

$6 *$ Correspondence to:

7 Lier Lin, No.19 Xiuhua Road, Hainan General Hospital, Haikou city, Hainan province, 570311, China. Email:

8 linlie123456202010@163.com

9

10

\section{Abstract}

Background: Acute myeloid leukemia (AML) is one of the most common blood cancers, and is characterized by impaired hematopoietic function and bone marrow (BM) failure. Under normal circumstances, autophagy may suppress tumorigenesis, however under the stressful conditions of late stage tumor growth autophagy actually protects tumor cells, so inhibiting autophagy in these cases also inhibits tumor growth and promotes tumor cell death.

Methods: AML gene expression profile data and corresponding clinical data were obtained from the Cancer Genome Atlas (TCGA) and Gene Expression Omnibus (GEO) databases, from which prognostic-related genes were screened to construct a risk score model through LASSO and univariate and multivariate Cox analyses. Then the model was verified in the TCGA cohort and GEO cohorts. In addition, we also analyzed the relationship between autophagy genes and immune infiltrating cells and therapeutic drugs.

Results: We built a model containing 10 autophagy-related genes to predict the survival of AML patients by dividing them into high- or low-risk subgroups. The high-risk subgroup was prone to a poorer prognosis in both the training TCGA-LAML cohort and the validation GSE37642 cohort. Univariate and multivariate Cox analysis revealed that the risk score of the autophagy model can be used as an independent prognostic factor. The high-risk subgroup had not only higher fractions 
27 of CD4 naïve T cell, NK cell activated, and resting mast cells but also higher expression of immune

28 checkpoint genes CTLA4 and CD274. Last, we screened drug sensitivity between high- and low-

29 risk subgroups.

30 Conclusion: The risk score model based on 10 autophagy-related genes can serve as an effective

31 prognostic predictor for AML patients and may guide for patient stratification for 32 immunotherapies and drugs.

33 Keywords: Acute myeloid leukemia, autophagy, TCGA, GEO, risk model 


\section{Introduction}

56

57

Acute myeloid leukemia (AML) is a kind of malignant blood cancer, accounting for about $1 \%$ of all cancers [1-3]. AML is characterized by impaired hematopoietic function and bone marrow (BM) failure, leading to fatal consequences due to the clonal expansion of undifferentiated myeloid progenitor cells [4-6]. Autophagy is an important biological process, vital to survival, differentiation, development, and homeostasis, and can play a very important role in tumors. Under normal circumstances, autophagy can inhibit the early development of cancer [7-10] by eliminating damaged proteins and organelles and reducing cell damage and chromosome instability. However, under hypoxic or low nutritional conditions, tumors can obtain nutrients through autophagy [11-14]. Recent studies found that inhibiting autophagy effectively inhibits tumor growth and promotes tumor cell death [15-17]. Moreover, autophagy-related gene signatures can effectively predict the clinical outcome of pancreatic ductal adenocarcinoma and breast tumors, but the research on autophagy prognostic biomarkers of AML is still insufficient. In this study, we used AML data from the TCGA database (TCGA-LAML) and the GEO database (GSE37642). We obtained 35 prognosis-related autophagy genes in the TCGA data and used 10 of those to construct a prognostic model and then verified it through the GEO database. Our model had good predictive performance suggests that these 10 autophagy genes may be related to the tumor microenvironment and could provide new insights for the therapeutic strategies and prognosis of AML.

\section{Materials and Methods}

\subsection{Database}

The TCGA-LAML dataset $(\mathrm{n}=200)$ was obtained from the TGCA database (https://portal.gdc.cancer.gov/). After deleting data with imperfect clinical information, we included the remaining 140 patients in the study. The GSE37642 dataset was obtained from the GEO database (https://www.ncbi.nlm.nih.gov/geo/query/acc.cgi?acc=GSE37642), and we specifically used the two datasets GSE37642-GPL96 and GSE37642-GPL570. After merging (n 
81 =562), we used "sva" R package to eliminate any batch effects [18-20]. The TCGA-LAML cohorts were the training group, the GSE37642 cohorts were the verification group. The autophagy gene set (Supplemental Table 1) was obtained from the autophagy database (http://www.autophagy.lu/).

\subsection{Autophagy Signature Construction and Validation}

Autophagy-related genes were extracted from TCGA-LAML, and univariate Cox analysis was used, with $\mathrm{p}<0.05$ considered significant. Next, we performed LASSO analysis and multivariate Cox to obtain the most critical prognostic genes, and then construct an autophagy model. The LASSO coefficients $(\beta)$ as follows:

Risk Score $=(\beta m R N A 1 \times$ expression level of mRNA1 $)+(\beta m R N A 2 \times$ expression level of mRNA2 $)$ $+\cdots+\left(\beta\right.$ mRNAn ${ }^{\times}$expression level of mRNAn $)[21-23]$.

The $\beta$ in this formula refers to the regression coefficient. The GSE37642 data set was used as a validation 1 cohort. In addition, we further verified the reliability of the prognostic gene signature by randomly dividing the training set (TCGA-LAML) into a verification 2 cohort and a verification 3 cohort. The autophagy risk score of each patient was calculated according to the uniform formula determined in the training cohort. We determine the best autophagy risk scoring standard through the "survminer" software package [24], and then divide the patients into high- and low-risk groups. In addition, we also constructed a prognostic nomogram.

\subsection{Estimation of immune cell type fractions}

The CIBERSORT algorithm is used to estimate the immune cell types of TCGA data [25-28].

\subsection{Generation of ImmuneScore and StromalScore}

The ESTIMATE package [29] was used to estimate the ratio of immune-stromal components in each sample in the tumor microenvironment in the form of two kinds of scores: Immune Score, and Stromal Score, which positively correlate with the ratio of immune and stroma, respectively. Meaning the higher the respective score, the larger the ratio of the corresponding component in the tumor microenvironment.

\subsection{Functional enrichment analysis}

The Kyoto Encyclopedia of Genes and Genomes (KEGG) and Gene Ontology (GO) analysis of 
108 all differentially expressed genes (DEGs) by $\mathrm{R}$ software with $p<0.01$ set as the threshold. Gene

109 Set Enrichment Analysis (GSEA software, version 4.0.1) was used to investigate the pathways

110 enriched in the high-risk subgroups. The number of random sample permutations was set at 10.

$111 \quad 2.6$ Statistical Analysis

112 LASSO analysis was performed using the "glmnet" package [30, 31]. The number of folds used in

113 cross-validation was 10 . The Time-dependent receiver operating characteristic (ROC) curve was

114 used to evaluate the predictive performance of 10 -gene features. The area under the ROC curve

115 (AUC) was calculated by using the "survivalROC" package [32-35]. The decision curve analysis

116 was carried out using the "rmda" software package. The "rms" software package was used for

117 nomogram and calibration diagrams. We use one-way ANOVA to analyze multiple sets of

118 normalized data. All statistical analyses were performed using R software (version 3.5.1) and

119 GraphPad Software (version 7.00). $p<0.05$ is considered statistically significant.

120 3. Results

$121 \quad 3.1$ Establishing an autophagy-related model and functional enrichment analysis

122 Thirty-five autophagy genes were related to prognosis in TCGA (Fig. 1A), and LASSO regression 123 analysis narrowed down the list (Fig. 1B, 1C), to include 10 autophagy genes (BAG3, BNIP3, $124 C A N X, C D K N 2 A, D I R A S 3, N R G 2, P A R P 1, P R K C D, V A M P 3, W D F Y 3)$ for prognostic model 125 construction (Fig. 1D).

126 The GO results indicated that 10 autophagy genes were significantly enriched in the biological 127 process (BP) and cellular components (CC) categories (Fig.1E), such as positive regulation of 128 protein localization to nucleus, regulation of muscle cell apoptotic process, muscle cell apoptotic 129 process, regulation of protein localization to nucleus, negative regulation of organelle 130 organization, positive regulation of muscle cell apoptotic process, positive regulation of protein 131 import into nucleus, positive regulation of protein import, intrinsic apoptotic signaling pathway in 132 response to oxidative stress, protein localization to nucleus, intrinsic apoptotic signaling pathway, 133 regulation of striated muscle cell apoptotic process, regulation of protein import into nucleus, 134 negative regulation of mitochondrion organization, striated muscle cell apoptotic process, selective 
135

136

137

138

139

140

141

142

143

144

145

146

147

148

149

150

151

152

153

154

155

156

157

158

159

160

161

autophagy, regulation of protein import, inclusion body, integral component of organelle membrane, intrinsic component of organelle membrane, and nuclear envelope. In addition, it is worth noting that the results of the KEGG analysis did not enrich for obvious pathways.

\subsection{Evaluation of autophagy risk score}

After dividing patients into high-risk and low-risk subgroups, we found an important result that the high-risk group was significantly associated with poor prognosis in the TCGA-LAML cohort $(P=6.975 \mathrm{e}-09 ;$ Fig. 2A). The AUC of the one-, three-, and five-year overall survival (OS) in the TCGA-LAML cohort were 0.819, 0.846, and 0.887, respectively (Fig. 2B). Compared with the other six signatures [36], our signature showed a higher C-index (0.7240) and AUCs for one-, three-, and five-year OS predictions (Fig. 2C, 2D).

In order to verify the predictive value of the 10-gene signature, we calculated the risk scores of patients in the GSE37642 cohort (validation 1 set). We found that the results of the GSE37642 cohort were consistent with the results in the TCGA cohort, and the OS of the high-risk group was significantly lower than that of the low-risk group $(P<0.001)$. The AUCs for one-, three-, and five-year OS were 0.638, 0.553, and 0.532, respectively (Fig. 2F). In addition, we further verified the reliability of the model. We randomly dividing the training set into a verification 2 set (Supplementary Fig. 1 A-D) and a verification 3 set (Supplementary Fig.1E-H), the signature had reliable predictive ability (Supplementary Fig. 1). Taking this together, the 10-gene signature was capable of predicting OS in AML. The clinical information of the patients was shown in Supplemental Table 2 .

\subsection{Clinical correlation analysis}

Univariate and multivariate COX analysis of clinically relevant factorsshowed that age $(p<0.001)$ and riskScore $(p<0.001)$ were independent prognostic indicators in the TCGA-LAML cohort (Fig. 3A, 3B), and that age $(p<0.001)$, runx1-mutation $(p<0.001)$, and riskScore $(p=0.019)$ were independent prognostic indicators in the GSE37642 cohort (Fig. 3C, 3D).

\subsection{Nomogram analysis results of TCGA-LAML cohort and GSE37642 cohort}

In order to better evaluate the relationship between genes and prognosis in the model, we used a 
162

163

164

165

166

167

168

169

170

171

172

173

174

175

176

177

178

179

180

181

182

183

184

185

186

187

188

nomogram to analyze it. The results show that in the TCGA-LAML cohort, BNIP3, CANX, and WDFY3 have a positive correlation with OS, and BAG3, CDKN2A, DIRAS3, NRG2, PARP1, PRKCD, and $V A M P 3$ have a negative correlation with OS (Fig.4A). In addition, in the GSE37642 cohort, $C A N X, C D K N 2 A, N R G 2$, and $V A M P 3$ have a positive correlation with OS, and $B A G 3$, BNIP3, DIRAS3, PARP1, PRKCD, and WDFY3 have a negative correlation with OS (Fig. 5A). The calibration plots showed that the nomogram could accurately predict the one-, three-, and fiveyear OS (Fig. 4B-D, Fig. 5B-D) with a harmonious consistency (TCGA-LAML, C-index = 0.72; GSE37642, C-index $=0.66$ ) between the predicted and observed survival.

\subsection{Significant differences between high- and low-risk subgroups}

The patients were scored by autophagy-related gene models, and the patients were divided into high- and low-risk groups based on the optimal score. Principal components analysis (PCA) supports the classification of AML patients into two subgroups (Fig. 6A). In order to further analyze the difference between the high-risk and low-risk subgroups, the ESTIMATE algorithm was used to analyze the TCGA-LAML tumor microenvironment. The results showed that high ImmuneScore was significantly associated with poor survival (Fig. 6B). Another important finding was that ImmuneScore and StromalScore were higher in the high-risk group (Fig. 6C). In addition, age was significantly correlated with both Immune Score and Stromal Score (Fig. 6D).

In order to explore the differences in immune infiltrating cells in the high- and low-risk subgroups, we used the CIBERSORT algorithm to analyze the composition of 22 immune cells in the TCGALAML cohort (Supplementary Figure 2) and analyzed the correlation between different immune infiltrating cells (Supplementary Figure 3). In addition, the difference in immune infiltrating cells between high and low-risk subgroups is shown in Fig. 6E. Further analysis showed that the high expression of mast cells resting was associated with a better prognosis and the NK cells activated with high expression was associated with a poor prognosis (Fig. 6F).

PDL1 (CD274) and CTLA4 play a very important role in the immunotherapy of AML. We found that the high-risk group had higher expression levels of PDL1 and CTLA4 (Fig.6G). GSEA analysis results showed that KEGG CHEMOKINE SIGNALING PATHWAY, KEGG CELL 
ADHESION MOLECULES CAMS, KEGG CYTOKINE CYTOKINE RECEPTOR INTERACTION, KEGG HEMATOPOIETIC CELL LINEAGE, and KEGG INTESTINAL IMMUNE NETWORK FOR IGA PROC were enriched in the high-risk group (Fig. 6H). The results of drug sensitivity analysis showed that there are significant differences between 24 chemotherapy drugs between high-risk and low-risk patients, which may provide help for personalized treatment of AML patients (Fig. 7).

\section{Discussion}

Autophagy has been shown to play an important role in the occurrence and development of tumors, especially in AML [37-40]. Targeting autophagy can overcome the chemoresistance of acute myeloid leukemia [41], granulocytic AML differentiation relies on non-canonical autophagy pathways, and restoring autophagic activity might be beneficial in differentiation therapies [4244]. CXCR4-mediated signal-regulated autophagy can also affect the survival and drug resistance of acute myeloid leukemia cells [45].

In this study, we first identified 10 autophagy genes related to AML patients' prognosis from the training group through univariate COX analysis, LASSO regression analysis, and multivariate COX analysis, to establish a risk score model. According to the optimal value of risk score, patients were divided into high- and low-risk subgroups. In the training group, a high-risk score was significantly correlated with poor prognosis $(p=6.975 \mathrm{e}-09)$. Then we conducted verification in the GSE37642 cohort, and the results supported that high-risk subgroups were significantly more related to poor prognosis $(p<0.001)$. Next, we tested the accuracy of the model, and the results showed that the predictive performance of the model was good (Fig. 2B, 2F). Interestingly, there was a tendency of shorter survival in patients with higher risks in TCGA data but not in GSE37642 (Fig. 2C, 2D, 2G, 2H). Testing with clinically relevant factors indicates that risk score in our model is an independent factor for AML in both TCGA-LAML and GSE37642 cohorts. Furthermore, the nomogram displayed the correlation between one-, three-, and five-year survival and these genes in the risk model. Among them, $C A N X, B A G 3, D I R A S 3, P A R P 1$, and $P R K C D$ are more consistent in both TCGA-LAML and GSE37642 cohorts. This is partly a reflection of the lower efficiency 
216

217

218

219

220

221

222

223

224

225

226

227

228

229

230

231

232

233

234

235

236

237

238

239

240

241

242

of TCGA-LAML cohort when compared to GSEA cohorts. Additional data could help validate and optimize the model.

In addition, we analyzed the relationship between autophagy genes and immune infiltrating cells in the model, and the results showed that the high-risk subgroup had a higher level of StromalScore, ImmuneScore, and certain immune cell types compared to the low-risk subgroup, indicating that the model might have a special immune signature. Moreover, the expression level of immune checkpoint genes (CTLA4 and CD274) in patients with higher risk was higher than low-risk subgroups, suggesting this model provides more information for immune therapies like stratifying patients who are more sensitive for CTLA4 and CD274 immune therapies. Consequently, we xplored the relationship between AML and tumor environment in the TCGALAML cohort. We found StromalScore could not predict prognosis but higher ImmuneScore had a slightly better survival while age is a significant factor that influencing Stromal Score and Immune Score in TCGA-LAML cohort. However, for mast cells resting and NK cells activating, subgroups with relatively high- or low level had a significant different survival. Those findings supported that AML patients might respond to immune therapies and our model might help their clinical applications. On the other hand, the pathway enrichment in high-risk subgroup in GSEA showed the top five enriched pathways - KEGG CHEMOKINE SIGNALING PATHWAY, KEGG CELL ADHESION MOLECULES CAMS, KEGG CYTOKINE CYTOKINE RECEPTOR INTERACTION, KEGG HEMATOPOIETIC CELL LINEAGE, and KEGG INTESTINAL IMMUNE NETWORK FOR IGA PROC. This together with the immune environment relationship, these results help clarify the interactions among autophagy and other signaling pathways in AML.

$D I R A S 3$, one important gene in our risk score model, is an imprinted tumor suppressor gene that also plays a very vital role in ovarian and breast cancer [46-48]. PRKCD is a pro-apoptotic kinase, and some miRNAs can regulate tumors by targeting PRKCD [49-51]. VAMP3 is a member of the vesicle-associated membrane protein (VAMP)/synaptobrevin family [52-55]. Consistent with these studies, our research shows that these genes are potential therapeutic targets for postoperative 
243

244

245

246

247

248

249

250

251

252

253

254

255

256

257

258

259

260

261

262

263

264

265

266

267

268

269

diseases caused by microglial activation.

However, this study has some limitations. First, our study is mainly based on TCGA data, and most of the patients are white or Asian and we should be cautious to extend our findings to patients of other races. Second, our study is a retrospective analysis, and prospective studies are necessary to verify the results. Third, the AML datasets do not have complete clinical information, which may reduce the statistical validity and reliability. Finally, verification of our model in vitro or in vivo would be beneficial.

Overall, we constructed a prognostic model of 10 autophagy-related genes through the TCGA database and verified them through the GEO database. Our results complement the existing prognostic models and can be used as potential biomarkers for AML. In addition, we provide new views on the role of autophagy genes in AML, and these autophagy genes may also be applied in clinical adjuvant therapy.

\section{Funding}

This work was supported by the Medical and health research projects in Hainan Province (Grant No. 2001320243A2009).

\section{Author's contribution}

Li Huang and Lier Lin designed the study, analyzed data, and wrote the manuscript. Lier Lin, Xiangjun Fu, Can Meng, and Li Huang analyzed data and contributed in writing the manuscript.

\section{Competing Interests}

The authors declare that there were no competing interests associated with the manuscript.

\section{Availability of data and materials}

The datasets generated and/or analyzed during the current study are available in the TCGA repository, https://portal.gdc.cancer.gov/; and GEO repository, https://www.ncbi.nlm.nih.gov/geo/query/acc.cgi?acc=GSE37642.

9. Ethics Approval and Consent to Participate

No ethics approval was required for this work. All utilized public data sets were generated by others who obtained ethical approval. 
270

271

272

273

274

275

276

277

278

279

280

281

282

283

284

285

286

287

288

289

290

291

292

293

294

295

296

\section{Abbreviations}

AML Acute myeloid leukemia

KEGG Kyoto Encyclopedia of Genes and Genomes

GO gene ontology

DEGs differentially expressed genes

FC fold change

GSEA gene set enrichment analysis

HR hazard ratio

ROC receiver operating characteristic

AUC area under the ROC curve

LAML Acute myeloid leukemia

LASSO least absolute shrinkage and selection operator

TCGA The Cancer Genome Atlas

GEO Gene Expression Omnibus

\section{References}

1. Molica M, Breccia M, Foa R, Jabbour E, Kadia TM. Maintenance therapy in AML: The past, the present and the future. Am J Hematol. 2019 Nov;94(11):1254-1265. doi: 10.1002/ajh.25620. Epub 2019 Sep 11. PMID: 31429099.

2. Winer ES, Stone RM. Novel therapy in Acute myeloid leukemia (AML): moving toward targeted approaches. Ther Adv Hematol. 2019 Jul 10;10:2040620719860645. doi: 10.1177/2040620719860645. PMID: 31321011; PMCID: PMC6624910.

3. Moors I, Vandepoele K, Philippé J, Deeren D, Selleslag D, Breems D, Straetmans N, Kerre T, Denys B. Clinical implications of measurable residual disease in AML: Review of current evidence. Crit Rev Oncol Hematol. 2019 Jan;133:142-148. doi: 10.1016/j.critrevonc.2018.11.010. Epub 2018 Nov 24. PMID: 30661650.

4. Cai SF, Levine RL. Genetic and epigenetic determinants of AML pathogenesis. Semin Hematol. 2019 Apr;56(2):84-89. doi: 10.1053/j.seminhematol.2018.08.001. Epub 2018 Aug 22. PMID: 30926095.

5. Hunter AM, Sallman DA. Current status and new treatment approaches in TP53 mutated AML. Best Pract 
297 Res Clin Haematol. 2019 Jun;32(2):134-144. doi: 10.1016/j.beha.2019.05.004. Epub 2019 May 11. PMID: 29831203995.

299 6. Gill SI. How close are we to CAR T-cell therapy for AML? Best Pract Res Clin Haematol. 2019 300 Dec;32(4):101104. doi: 10.1016/j.beha.2019.101104. Epub 2019 Oct 18. PMID: 31779970.

301 7. Onorati AV, Dyczynski M, Ojha R, Amaravadi RK. Targeting autophagy in cancer. Cancer. 2018 302 Aug;124(16):3307-3318. doi: 10.1002/cncr.31335. Epub 2018 Apr 19. PMID: 29671878; PMCID: PMC6108917.

8. Glick D, Barth S, Macleod KF. Autophagy: cellular and molecular mechanisms. J Pathol. 2010 May;221(1):312. doi: 10.1002/path.2697. PMID: 20225336; PMCID: PMC2990190.

9. Mizushima N, Komatsu M. Autophagy: renovation of cells and tissues. Cell. 2011 Nov 11;147(4):728-41. doi: 10.1016/j.cell.2011.10.026. PMID: 22078875.

10. Li YJ, Lei YH, Yao N, Wang CR, Hu N, Ye WC, Zhang DM, Chen ZS. Autophagy and multidrug resistance in cancer. Chin J Cancer. 2017 Jun 24;36(1):52. doi: 10.1186/s40880-017-0219-2. PMID: 28646911; PMCID: PMC5482965.

311 11. Boya P, Esteban-Martínez L, Serrano-Puebla A, Gómez-Sintes R, Villarejo-Zori B. Autophagy in the eye:

312 Development, degeneration, and aging. Prog Retin Eye Res. 2016 Nov;55:206-245. doi: 313 10.1016/j.preteyeres.2016.08.001. Epub 2016 Aug 23. PMID: 27566190.

314 12. Kim KH, Lee MS. Autophagy--a key player in cellular and body metabolism. Nat Rev Endocrinol. 2014 315 Jun;10(6):322-37. doi: 10.1038/nrendo.2014.35. Epub 2014 Mar 25. PMID: 24663220.

316 13. Fan Z, Liu H, Xue Y, Lin J, Fu Y, Xia Z, Pan D, Zhang J, Qiao K, Zhang Z, Liao Y. Reversing cold tumors 317 to hot: An immunoadjuvant-functionalized metal-organic framework for multimodal imaging-guided synergistic 318 photo-immunotherapy. Bioact Mater. 2020 Aug 28;6(2):312-325. doi: 10.1016/j.bioactmat.2020.08.005. PMID: 319 32954050; PMCID: PMC7475520.

320 14. Parzych KR, Klionsky DJ. An overview of autophagy: morphology, mechanism, and regulation. Antioxid 321 Redox Signal. 2014 Jan 20;20(3):460-73. doi: 10.1089/ars.2013.5371. Epub 2013 Aug 2. PMID: 23725295; 322 PMCID: PMC3894687.

323 15. Luan F, Chen W, Chen M, Yan J, Chen H, Yu H, Liu T, Mo L. An autophagy-related long non-coding RNA 
324

325

326

327

328

329

330

331

332

333

334

335

336

337

338

339

340

341

342

343

344

345

346

347

348

349

350

signature for glioma. FEBS Open Bio. 2019 Mar 5;9(4):653-667. doi: 10.1002/2211-5463.12601. PMID: 30984540; PMCID: PMC6443865.

16. Wang Z, Gao L, Guo X, Feng C, Lian W, Deng K, Xing B. Development and validation of a nomogram with an autophagy-related gene signature for predicting survival in patients with glioblastoma. Aging (Albany NY). 2019 Dec 17;11(24):12246-12269. doi: 10.18632/aging.102566. Epub 2019 Dec 17. PMID: 31844032; PMCID: PMC6949068.

17. Liang C, Xu J, Meng Q, Zhang B, Liu J, Hua J, Zhang Y, Shi S, Yu X. TGFB1-induced autophagy affects the pattern of pancreatic cancer progression in distinct ways depending on SMAD4 status. Autophagy. 2020 Mar;16(3):486-500. doi: 10.1080/15548627.2019.1628540. Epub 2019 Jun 17. PMID: 31177911; PMCID: PMC6999639.

18. Varma S. Blind estimation and correction of microarray batch effect. PLoS One. 2020 Apr 9;15(4):e0231446. doi: 10.1371/journal.pone.0231446. PMID: 32271844; PMCID: PMC7145015.

19. Leek JT, Storey JD. Capturing heterogeneity in gene expression studies by surrogate variable analysis. PLoS Genet. 2007 Sep;3(9):1724-35. doi: 10.1371/journal.pgen.0030161. Epub 2007 Aug 1. PMID: 17907809; PMCID: PMC1994707.

20. Leek JT, Johnson WE, Parker HS, Jaffe AE, Storey JD. The sva package for removing batch effects and other unwanted variation in high-throughput experiments. Bioinformatics. 2012 Mar 15;28(6):882-3. doi: 10.1093/bioinformatics/bts034. Epub 2012 Jan 17. PMID: 22257669; PMCID: PMC3307112.

21. Livingston MJ, Ding HF, Huang S, Hill JA, Yin XM, Dong Z. Persistent activation of autophagy in kidney tubular cells promotes renal interstitial fibrosis during unilateral ureteral obstruction. Autophagy. 2016 Jun 2;12(6):976-98. doi: 10.1080/15548627.2016.1166317. Epub 2016 Apr 28. PMID: 27123926; PMCID: PMC4922446.

22. Apfel CC, Läärä E, Koivuranta M, Greim CA, Roewer N. A simplified risk score for predicting postoperative nausea and vomiting: conclusions from cross-validations between two centers. Anesthesiology. 1999 Sep;91(3):693-700. doi: 10.1097/00000542-199909000-00022. PMID: 10485781.

23. Toulopoulou T, Zhang X, Cherny S, Dickinson D, Berman KF, Straub RE, Sham P, Weinberger DR. Polygenic risk score increases schizophrenia liability through cognition-relevant pathways. Brain. 2019 Feb 
351

352

353

354

355

356

357

358

359

360

361

362

363

364

365

366

367

368

369

370

371

372

373

374

375

376

377

1;142(2):471-485. doi: 10.1093/brain/awy279. PMID: 30535067; PMCID: PMC6359897.

24. Walter W, Sánchez-Cabo F, Ricote M. GOplot: an R package for visually combining expression data with functional analysis. Bioinformatics. 2015 Sep 1;31(17):2912-4. doi: 10.1093/bioinformatics/btv300. Epub 2015 May 11. PMID: 25964631.

25. Alaa AM, Bolton T, Di Angelantonio E, Rudd JHF, van der Schaar M. Cardiovascular disease risk prediction using automated machine learning: A prospective study of 423,604 UK Biobank participants. PLoS One. 2019 May 15;14(5):e0213653. doi: 10.1371/journal.pone.0213653. PMID: 31091238; PMCID: PMC6519796.

26. Gentles AJ, Newman AM, Liu CL, Bratman SV, Feng W, Kim D, Nair VS, Xu Y, Khuong A, Hoang CD, Diehn M, West RB, Plevritis SK, Alizadeh AA. The prognostic landscape of genes and infiltrating immune cells across human cancers. Nat Med. 2015 Aug;21(8):938-945. doi: 10.1038/nm.3909. Epub 2015 Jul 20. PMID: 26193342; PMCID: PMC4852857.

27. Newman AM, Steen CB, Liu CL, Gentles AJ, Chaudhuri AA, Scherer F, Khodadoust MS, Esfahani MS, Luca BA, Steiner D, Diehn M, Alizadeh AA. Determining cell type abundance and expression from bulk tissues with digital cytometry. Nat Biotechnol. 2019 Jul;37(7):773-782. doi: 10.1038/s41587-019-0114-2. Epub 2019 May 6. PMID: 31061481; PMCID: PMC6610714.

28. Chen B, Khodadoust MS, Liu CL, Newman AM, Alizadeh AA. Profiling Tumor Infiltrating Immune Cells with CIBERSORT. Methods Mol Biol. 2018;1711:243-259. doi: 10.1007/978-1-4939-7493-1_12. PMID: 29344893; PMCID: PMC5895181.

29. Yoshihara K, Shahmoradgoli M, Martínez E, Vegesna R, Kim H, Torres-Garcia W, Treviño V, Shen H, Laird PW, Levine DA, Carter SL, Getz G, Stemke-Hale K, Mills GB, Verhaak RG. Inferring tumour purity and stromal and immune cell admixture from expression data. Nat Commun. 2013;4:2612. doi: 10.1038/ncomms3612. PMID: 24113773; PMCID: PMC3826632.

30. Engebretsen S, Bohlin J. Statistical predictions with glmnet. Clin Epigenetics. 2019 Aug 23;11(1):123. doi: 10.1186/s13148-019-0730-1. PMID: 31443682; PMCID: PMC6708235.

31. Blanco JL, Porto-Pazos AB, Pazos A, Fernandez-Lozano C. Prediction of high anti-angiogenic activity peptides in silico using a generalized linear model and feature selection. Sci Rep. 2018 Oct 24;8(1):15688. doi: 10.1038/s41598-018-33911-z. PMID: 30356060; PMCID: PMC6200741. 
32. Le NQK, Do DT, Hung TNK, Lam LHT, Huynh TT, Nguyen NTK. A Computational Framework Based on Ensemble Deep Neural Networks for Essential Genes Identification. Int J Mol Sci. 2020 Nov 28;21(23):9070. doi: 10.3390/ijms21239070. PMID: 33260643; PMCID: PMC7730808.

33. Do DT, Le NQK. Using extreme gradient boosting to identify origin of replication in Saccharomyces cerevisiae via hybrid features. Genomics. 2020 May;112(3):2445-2451. doi: 10.1016/j.ygeno.2020.01.017. Epub 2020 Jan 24. PMID: 31987913.

34. Li M, Shang H, Wang T, Yang SQ, Li L. Huanglian decoction suppresses the growth of hepatocellular carcinoma cells by reducing CCNB1 expression. World J Gastroenterol. 2021 Mar 14;27(10):939-958. doi: 10.3748/wjg.v27.i10.939. PMID: 33776365; PMCID: PMC7968131.

35. Le NQK, Ho QT, Nguyen TT, Ou YY. A transformer architecture based on BERT and 2D convolutional neural network to identify DNA enhancers from sequence information. Brief Bioinform. 2021 Feb 5:bbab005. doi: 10.1093/bib/bbab005. Epub ahead of print. PMID: 33539511.

36. Chen XX, Li ZP, Zhu JH, Xia HT, Zhou H. Systematic Analysis of Autophagy-Related Signature Uncovers Prognostic Predictor for Acute Myeloid Leukemia. DNA Cell Biol. 2020 Sep;39(9):1595-1605. doi: 10.1089/dna.2020.5667. Epub 2020 Aug 12. PMID: 32783661; PMCID: PMC7482110.

37. Yun CW, Lee SH. The Roles of Autophagy in Cancer. Int J Mol Sci. 2018 Nov 5;19(11):3466. doi: 10.3390/ijms19113466. PMID: 30400561; PMCID: PMC6274804.

38. Fan Z, Xiao K, Lin J, Liao Y, Huang X. Functionalized DNA Enables Programming Exosomes/Vesicles for Tumor Imaging and Therapy. Small. 2019 Nov;15(47):e1903761. doi: 10.1002/smll.201903761. Epub 2019 Oct 15. PMID: 31614072.

39. Levy JMM, Towers CG, Thorburn A. Targeting autophagy in cancer. Nat Rev Cancer. 2017 Sep;17(9):528542. doi: 10.1038/nrc.2017.53. Epub 2017 Jul 28. PMID: 28751651 ; PMCID: PMC5975367.

40. Zhang F, Li J, Zhu J, Liu L, Zhu K, Cheng S, Lv R, Zhang P. IRF2-INPP4B-mediated autophagy suppresses apoptosis in acute myeloid leukemia cells. Biol Res. 2019 Mar 15;52(1):11. doi: 10.1186/s40659-019-0218-7. PMID: 30876449; PMCID: PMC6419480.

41. Piya S, Andreeff M, Borthakur G. Targeting autophagy to overcome chemoresistance in acute myleogenous leukemia. Autophagy. 2017 Jan 2;13(1):214-215. doi: 10.1080/15548627.2016.1245263. Epub 2016 Oct 31.

Peer) reviewing PDF | (2021:04:60225:2:0:NEW 14 Jul 2021) 
PMID: 27797294; PMCID: PMC5240828.

42. Wu SY, Wen YC, Ku CC, Yang YC, Chow JM, Yang SF, Lee WJ, Chien MH. Penfluridol triggers cytoprotective autophagy and cellular apoptosis through ROS induction and activation of the PP2A-modulated MAPK pathway in acute myeloid leukemia with different FLT3 statuses. J Biomed Sci. 2019 Aug 31;26(1):63. doi: 10.1186/s12929-019-0557-2. PMID: 31470848; PMCID: PMC6717358.

43. San José-Enériz E, Gimenez-Camino N, Agirre X, Prosper F. HDAC Inhibitors in Acute Myeloid Leukemia. Cancers (Basel). 2019 Nov 14;11(11):1794. doi: 10.3390/cancers11111794. PMID: 31739588; PMCID: PMC6896008.

44. Jin J, Britschgi A, Schläfli AM, Humbert M, Shan-Krauer D, Batliner J, Federzoni EA, Ernst M, Torbett BE, Yousefi S, Simon HU, Tschan MP. Low Autophagy (ATG) Gene Expression Is Associated with an Immature AML Blast Cell Phenotype and Can Be Restored during AML Differentiation Therapy. Oxid Med Cell Longev. 2018 Mar 18;2018:1482795. doi: 10.1155/2018/1482795. PMID: 29743969; PMCID: PMC5878891.

45. Hu X, Mei S, Meng W, Xue S, Jiang L, Yang Y, Hui L, Chen Y, Guan MX. CXCR4-mediated signaling regulates autophagy and influences acute myeloid leukemia cell survival and drug resistance. Cancer Lett. 2018 Jul 1;425:1-12. doi: 10.1016/j.canlet.2018.03.024. Epub 2018 Mar 21. PMID: 29574276.

46. Sutton MN, Huang GY, Liang X, Sharma R, Reger AS, Mao W, Pang L, Rask PJ, Lee K, Gray JP, Hurwitz AM, Palzkill T, Millward SW, Kim C, Lu Z, Bast RC Jr. DIRAS3-Derived Peptide Inhibits Autophagy in Ovarian Cancer Cells by Binding to Beclin1. Cancers (Basel). 2019 Apr 18;11(4):557. doi: 10.3390/cancers11040557. PMID: 31003488; PMCID: PMC6521623.

47. Peng Y, Jia J, Jiang Z, Huang D, Jiang Y, Li Y. Oncogenic DIRAS3 promotes malignant phenotypes of glioma by activating EGFR-AKT signaling. Biochem Biophys Res Commun. 2018 Oct 28;505(2):413-418. doi: 10.1016/j.bbrc.2018.09.119. Epub 2018 Sep 25. PMID: 30266404.

48. Sutton MN, Lu Z, Li YC, Zhou Y, Huang T, Reger AS, Hurwitz AM, Palzkill T, Logsdon C, Liang X, Gray JW, Nan X, Hancock J, Wahl GM, Bast RC Jr. DIRAS3 (ARHI) Blocks RAS/MAPK Signaling by Binding Directly to RAS and Disrupting RAS Clusters. Cell Rep. 2019 Dec 10;29(11):3448-3459.e6. doi: 10.1016/j.celrep.2019.11.045. PMID: 31825828; PMCID: PMC6948147.

49. Zhang D, Xu X, Dong Z. PRKCD/PKC $\delta$ contributes to nephrotoxicity during cisplatin chemotherapy by 
432

433

434

435

436

437

438

439

440

441

442

456

457

458

56

suppressing autophagy. Autophagy. 2017 Mar 4;13(3):631-632. doi: 10.1080/15548627.2016.1269990. Epub 2017 Jan 6. PMID: 28059582; PMCID: PMC5361607.

50. Yao L, Wang L, Li F, Gao X, Wei X, Liu Z. MiR181c inhibits ovarian cancer metastasis and progression by targeting PRKCD expression. Int J Clin Exp Med. 2015 Sep 15;8(9):15198-205. PMID: 26629004; PMCID: PMC4658893.

51. Ke G, Liang L, Yang JM, Huang X, Han D, Huang S, Zhao Y, Zha R, He X, Wu X. MiR-181a confers resistance of cervical cancer to radiation therapy through targeting the pro-apoptotic PRKCD gene. Oncogene. 2013 Jun 20;32(25):3019-27. doi: 10.1038/onc.2012.323. Epub 2012 Jul 30. PMID: 22847611.

52. Sneeggen M, Pedersen NM, Campsteijn C, Haugsten EM, Stenmark H, Schink KO. WDFY2 restrains matrix metalloproteinase secretion and cell invasion by controlling VAMP3-dependent recycling. Nat Commun. 2019 Jun 28;10(1):2850. doi: 10.1038/s41467-019-10794-w. PMID: 31253801; PMCID: PMC6599030.

53. Chen Y, Sun JX, Chen WK, Wu GC, Wang YQ, Zhu KY, Wang J. miR-124/VAMP3 is a novel therapeutic target for mitigation of surgical trauma-induced microglial activation. Signal Transduct Target Ther. 2019 Aug 16;4:27. doi: 10.1038/s41392-019-0061-x. PMID: 31637007; PMCID: PMC6799846.

54. Pontes ER, Matos LC, da Silva EA, Xavier LS, Diaz BL, Small IA, Reis EM, Verjovski-Almeida S, Barcinski MA, Gimba ER. Auto-antibodies in prostate cancer: humoral immune response to antigenic determinants coded by the differentially expressed transcripts FLJ23438 and VAMP3. Prostate. 2006 Oct 1;66(14):1463-73. doi: 10.1002/pros.20439. PMID: 16897729.

55. Caronni N, Simoncello F, Stafetta F, Guarnaccia C, Ruiz-Moreno JS, Opitz B, Galli T, Proux-Gillardeaux V, Benvenuti F. Downregulation of Membrane Trafficking Proteins and Lactate Conditioning Determine Loss of Dendritic Cell Function in Lung Cancer. Cancer Res. 2018 Apr 1;78(7):1685-1699. doi: 10.1158/00085472.CAN-17-1307. Epub 2018 Jan 23. PMID: 29363545. 
459

460

461

462

463

464

465

466

467

468

469

470

471

472

473

474

475

476

477

478

479

480

481

482

483

484

485

\section{Figure legends:}

Fig.1 Construction of the autophagy model. (A) Univariate Cox analysis results of the TCGALAML cohort. (B) LASSO coefficients of autophagy-related genes. Each curve represents an autophagy gene. (C) 1,000-fold cross-validation for variable selection in the LASSO regression via 1-SE criteria. (D) Multivariate Cox analysis results. (E) GO analysis results.

Fig.2 Evaluation of Autophagy Risk Score. Kaplan-Meier curve of the prognostic model in the TCGA-LAML cohort (A) and GSE37642 cohort (E). Time-dependent ROC analysis for one-, three-, and five-year overall survival (OS) of a prognostic model in the TCGA-LAML cohort (B) and GSE37642 cohort (F). The distribution of the survival status of patients in the TCGA-LAML cohort (C) and GSE37642 cohort (G). The distribution of risk score in survival outcome analysis for TCGA-LAML cohort (D) and GSE37642 cohort (H).

Fig.3 Clinical correlation analysis. Forest plot of the univariate (left) and multivariate (right) Cox regression analysis in the TCGA-LAML cohort (A, B), and GSE37642 cohort (C, D) for Acute myeloid leukemia (AML).

Fig.4 Construction of a nomogram based on the 10 hub genes. (A) Construction of the nomogram in the TCGA cohort. (B-D) Calibration maps used to predict the 1-year (B), 3-year (C), and 5-year survival (D).

Fig.5 Validation of a nomogram based on the 10 hub genes. (A) Validation of the nomogram in the GSE37642 cohort. (B-D) Calibration maps used to predict the one -year (B), three -year (C), and five -year survival (D).

Fig.6 Analysis of differences between high- and low-risk subgroups (tumor microenvironment, immune cell infiltration, immune checkpoint regulators, and GSEA analysis). (A) PCA analysis supported the stratification into two AML subclasses (high-risk (red) and low-risk (blue) groups) in TCGA cohort. (B) The survival for subgroups with different stromalscore (left) and immunescore (right). (C) The high-risk group has a higher ImmuneScore and StromalScore. (D) Age has a significant correlation with both ImmuneScore and StromalScore. (E) The comparison of immune cell fractions between high- and low-risk subgroups. (F) A high-level of Mast cells 
486 resting is significantly associated with better survival, a high-level NK cells activated is 487 significantly associated with poor survival. (G) CTLA4 and CD274 have higher expression levels 488 in the high-risk group. (H) The pathways enriched in the high-risk group through GSEA analysis. 489 Fig.7 Drug sensitivity analysis to drugs of high- and low-risk subgroups.

490 Supplemental Figure 1 Signature verification. The training set randomly divide into a verification 2 491 set (Supplementary Fig.1 A-D) and a verification 3 set (Supplementary Fig.1E-H).

492 Supplemental Figure 2 The CIBERSORT to evaluate the composition of 22 immune cells in the 493 training group.

494 Supplemental Figure 3 The correlation between different immune infiltrating cells.

495 Supplemental Table 1 Autophagy-related genes.

496 Supplemental Table 2 The clinical information of the patients. 
Figure 1

Construction of the autophagy model.

(A) Univariate Cox analysis results of the TCGA-LAML cohort. (B) LASSO coefficients of autophagy-related genes. Each curve represents an autophagy gene. (C) 1,000-fold crossvalidation for variable selection in the LASSO regression via 1-SE criteria. (D) Multivariate Cox analysis results. (E) GO analysis results.

A

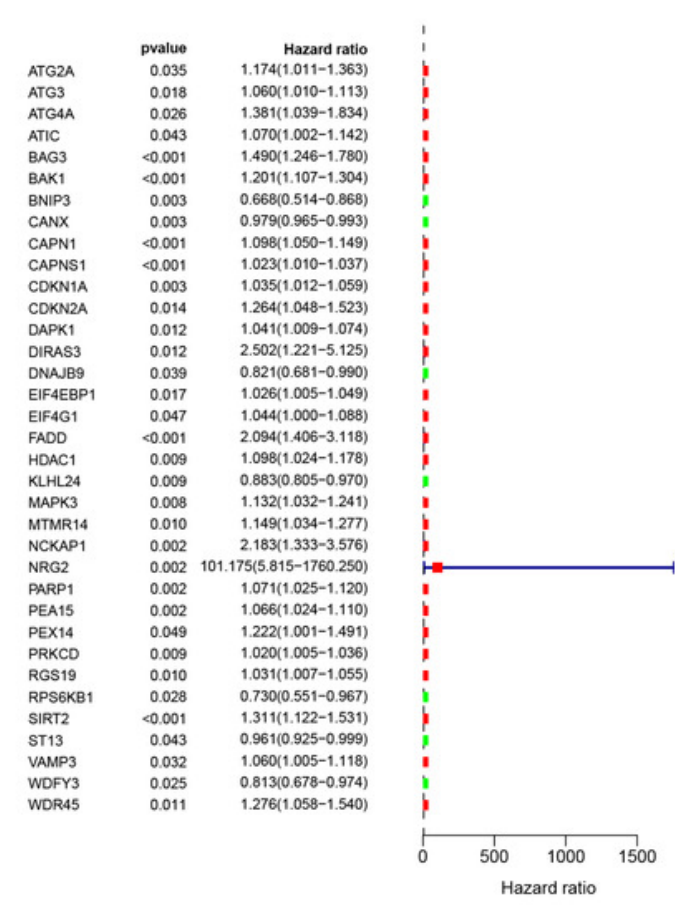

$\mathrm{E}$

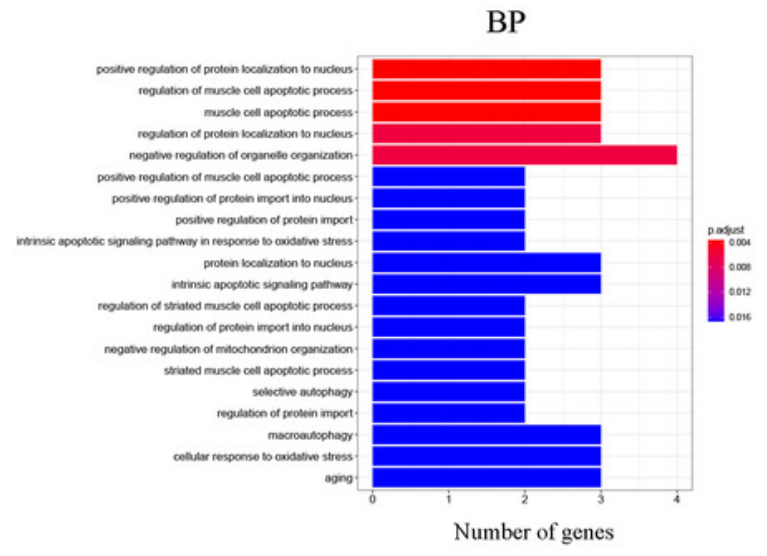

B

D
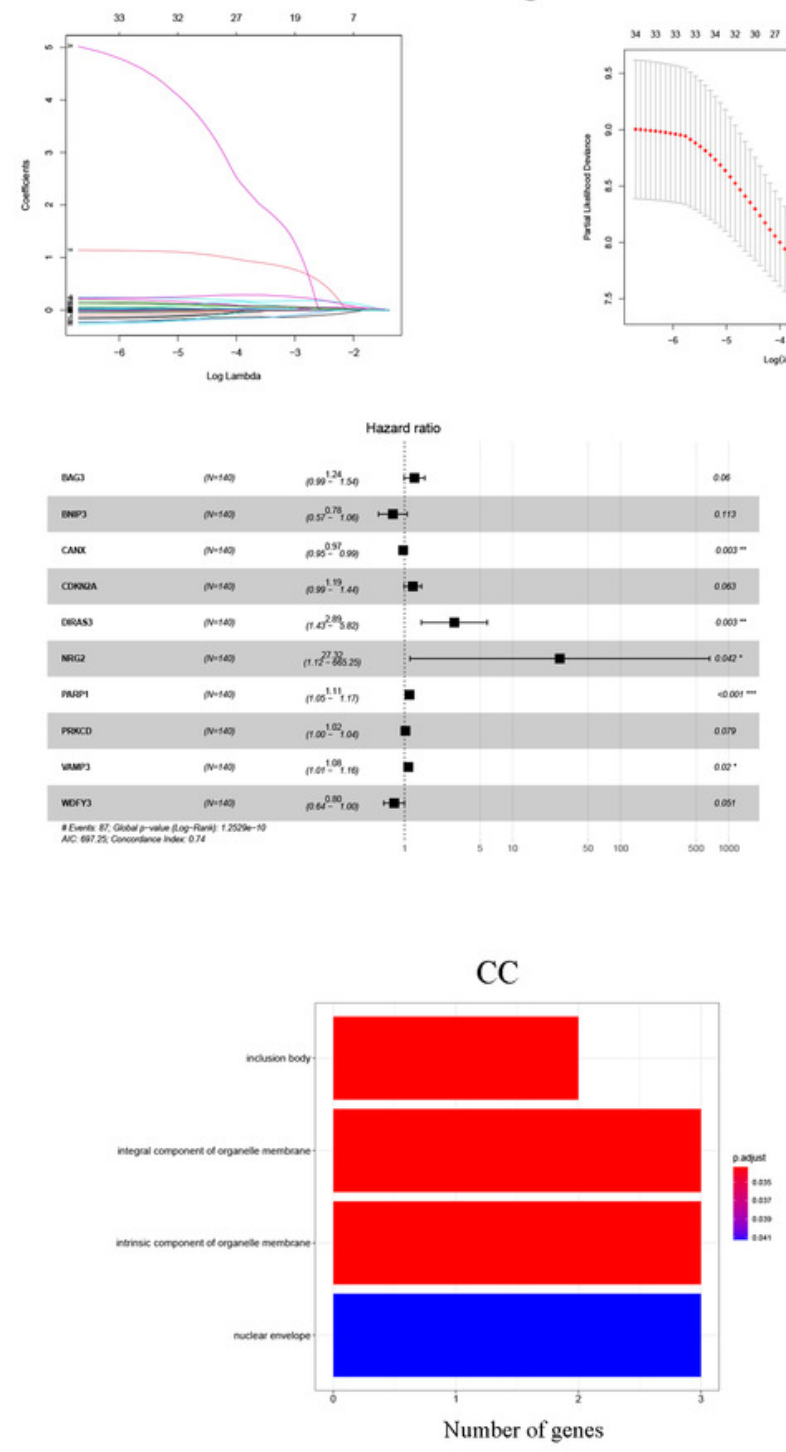


\section{Figure 2}

Evaluation of Autophagy Risk Score.

Kaplan-Meier curve of the prognostic model in the TCGA-LAML cohort (A) and GSE37642 cohort (E). Time-dependent ROC analysis for one-, three-, and five-year overall survival (OS) of a prognostic model in the TCGA-LAML cohort (B) and GSE37642 cohort (F). The distribution of the survival status of patients in the TCGA-LAML cohort (C) and GSE37642 cohort (G). The distribution of risk score in survival outcome analysis for TCGA-LAML cohort (D) and GSE37642 cohort (H).

A
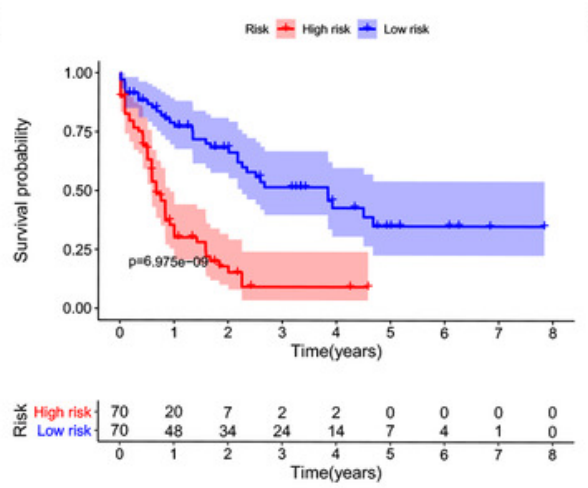

E

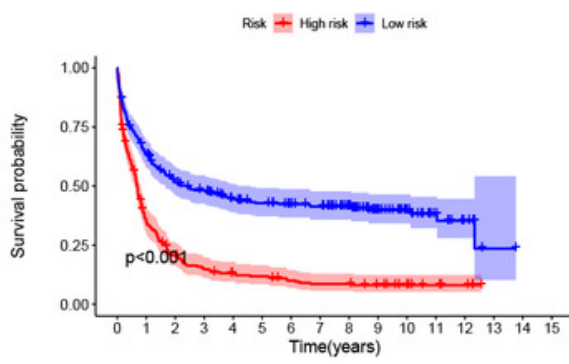

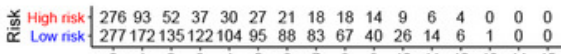

B

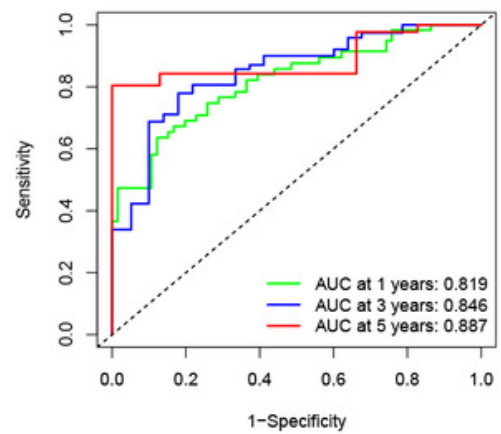

F

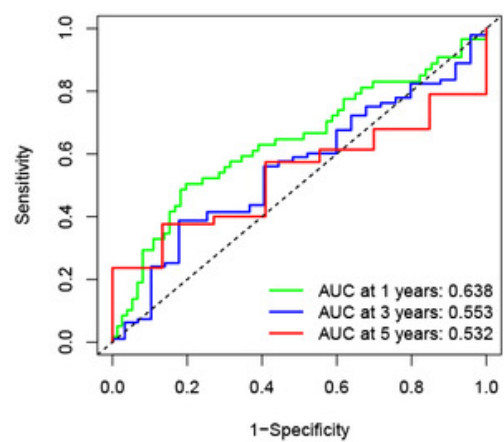

C

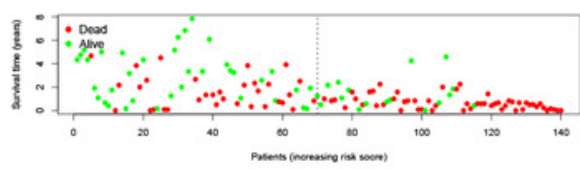

D

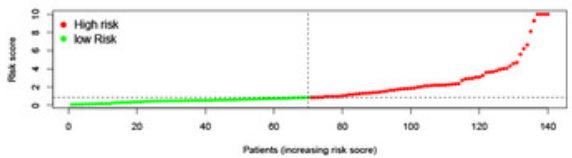

G

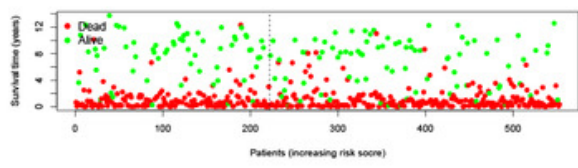

H

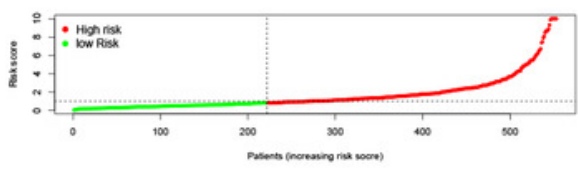


Figure 3

Clinical correlation analysis.

Forest plot of the univariate (left) and multivariate (right) Cox regression analysis in the TCGA-LAML cohort (A, B), and GSE37642 cohort (C, D) for Acute myeloid leukemia (AML).

A

pvalue Hazard ratio

age $\quad<0.001 \quad 1.042(1.026-1.058)$

gender $\quad 0.892 \quad 1.030(0.674-1.572)$

riskScore $<0.001 \quad 1.228(1.165-1.295)$

$$
\text { riskScore }<0.001 \quad 1.228(1.165-1.295)
$$

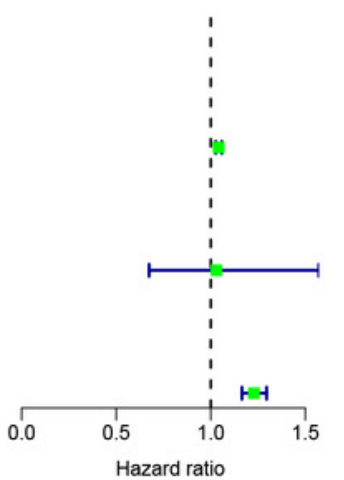

C

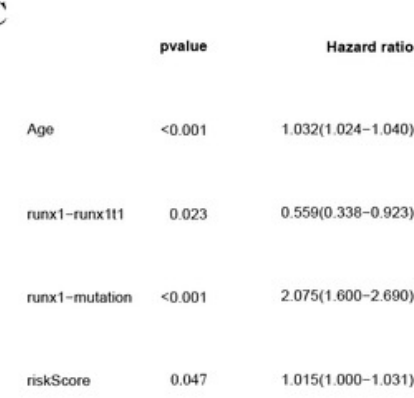

B

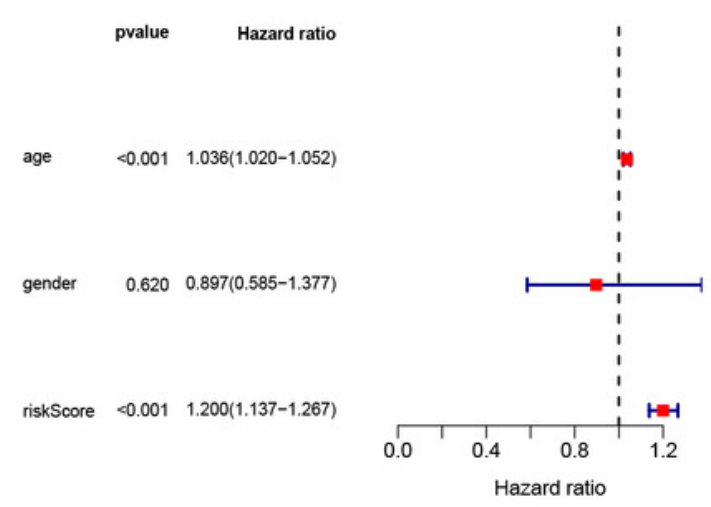

D

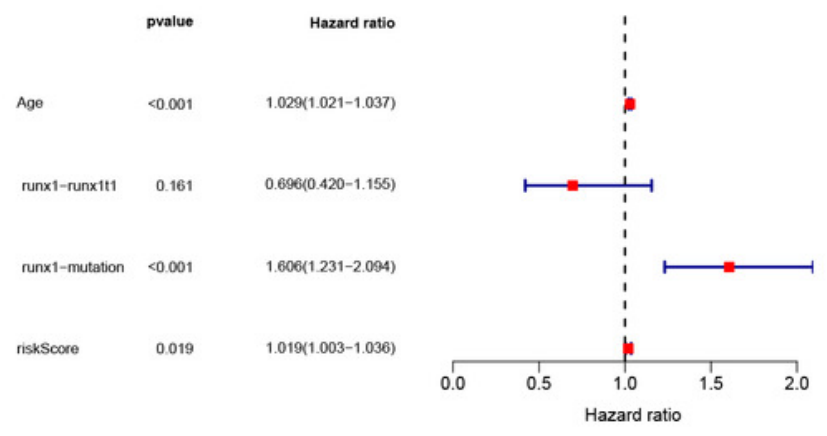


Figure 4

Construction of a nomogram based on the 10 hub genes.

(A) Construction of the nomogram in the TCGA cohort. (B-D) Calibration maps used to predict the 1-year (B), 3-year (C), and 5-year survival (D).

A

Points

BAG3

BNIP3

CANX

CDKN2A

DIRAS3

NRG2

PARP1

PRKCD

VAMP3

WDFY3

Total Points

1-year survival

3-year survival

5-year survival

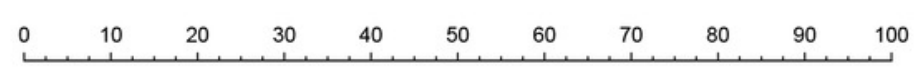

B

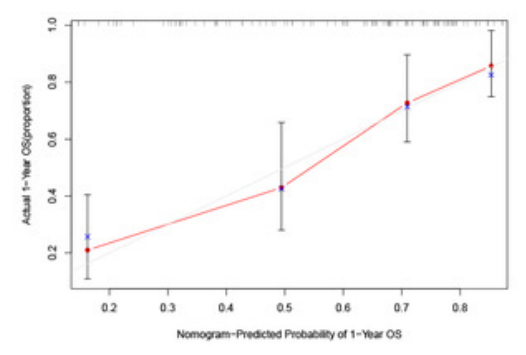

C

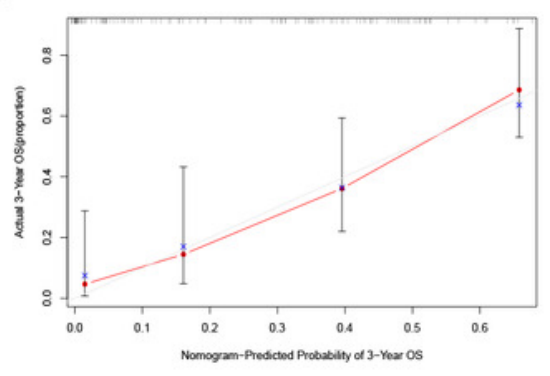

D

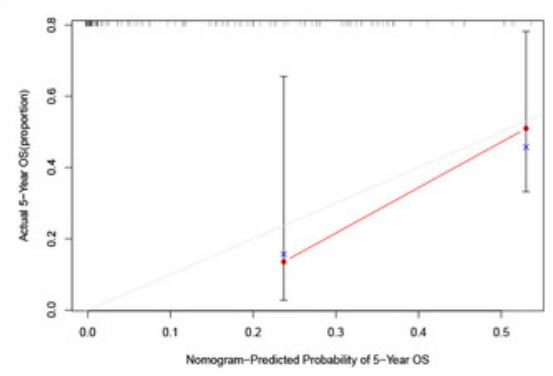


Figure 5

Validation of a nomogram based on the 10 hub genes.

(A) Validation of the nomogram in the GSE37642 cohort. (B-D) Calibration maps used to predict the one -year (B), three -year (C), and five -year survival (D).

A

Points

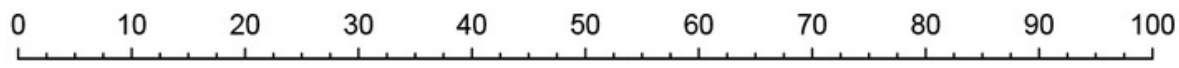

BAG3

BNIP3

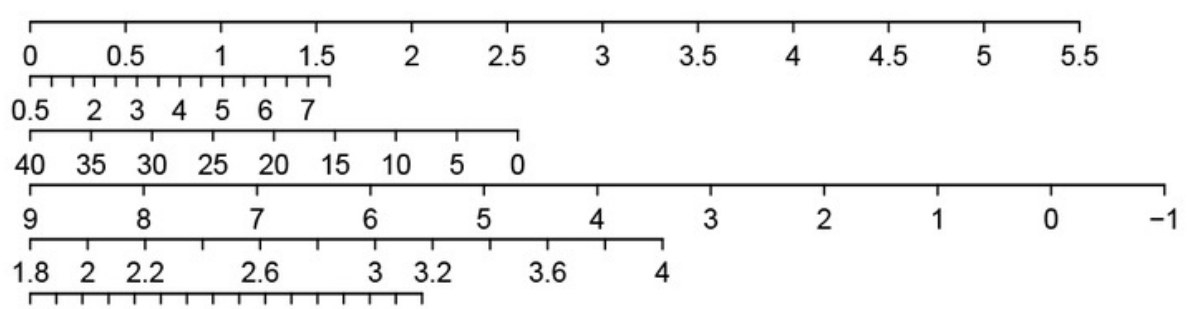

CANX

CDKN2A

DIRAS3

NRG2

\begin{tabular}{llllll}
2.7 & 2.55 & 2.4 & 2.25 & 2.1 & 2 \\
\hline
\end{tabular}

PARP1

PRKCD

VAMP3

WDFY3

Total Points

1-year survival

3-year survival

5-year survival

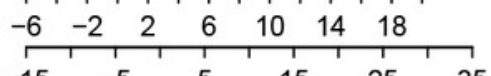

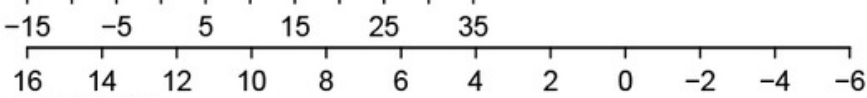

तागाताता

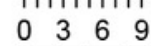

\begin{tabular}{|c|c|c|c|c|c|c|c|}
\hline \multirow[t]{4}{*}{0} & 50 & 100 & 150 & 200 & 250 & 300 & 350 \\
\hline & & & 0.8 & 0.7 & 0.5 & 0.3 & \\
\hline & & & 0.7 & 0.5 & 0.3 & 0.1 & \\
\hline & & & & 0.5 & 0.3 & 0.1 & \\
\hline
\end{tabular}

B

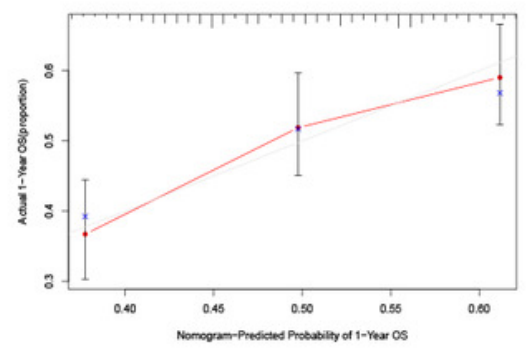

C

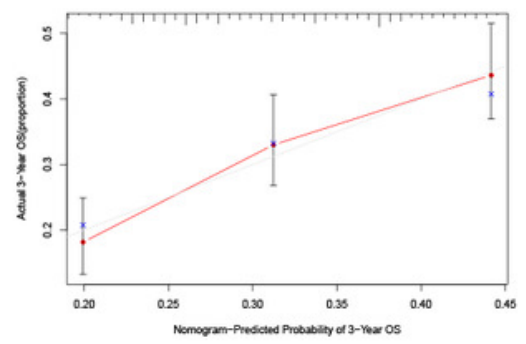

D

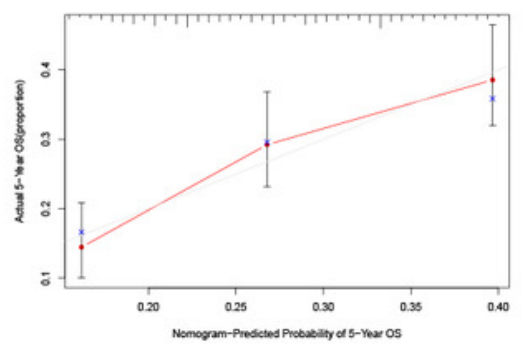




\section{Figure 6}

Analysis of differences between high- and low-risk subgroups (tumor microenvironment, immune cell infiltration, immune checkpoint regulators, and GSEA analysis).

(A) PCA analysis supported the stratification into two AML subclasses (high-risk (red) and lowrisk (blue) groups) in TCGA cohort. (B) The survival for subgroups with different stromalscore (left) and immunescore (right). (C) The high-risk group has a higher ImmuneScore and StromalScore. (D) Age has a significant correlation with both ImmuneScore and StromalScore. (E) The comparison of immune cell fractions between high- and low-risk subgroups. (F) A high-level of Mast cells resting is significantly associated with better survival, a high-level NK cells activated is significantly associated with poor survival. (G) CTLA4 and CD274 have higher expression levels in the high-risk group. (H) The pathways enriched in the high-risk group through GSEA analysis. 


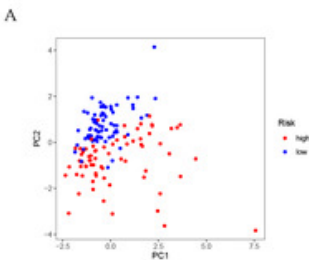

D
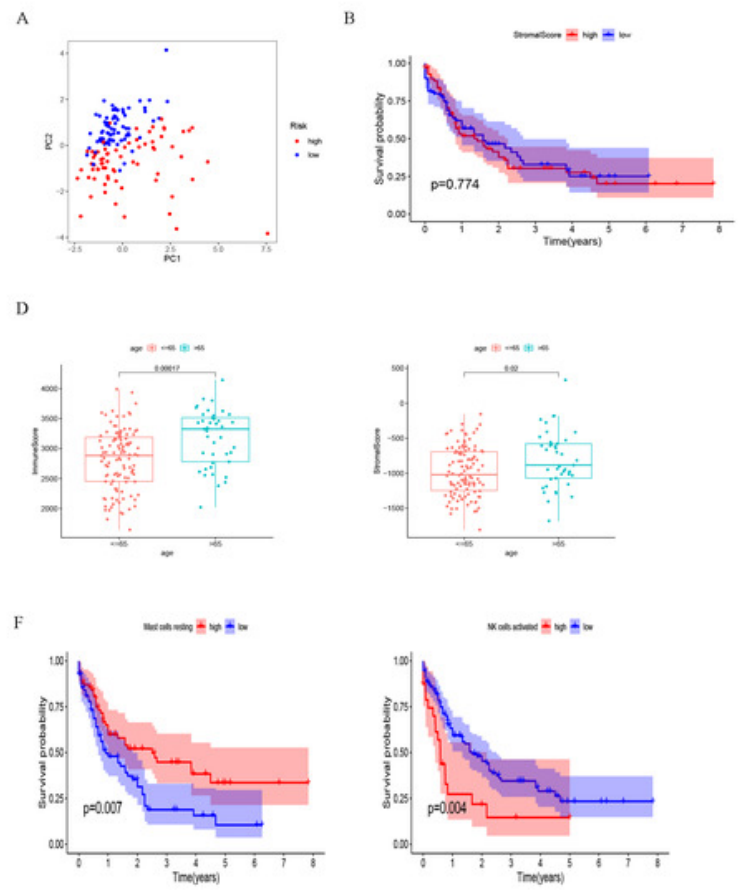

E

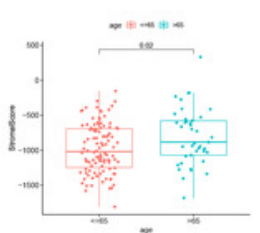

G

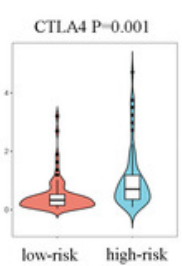

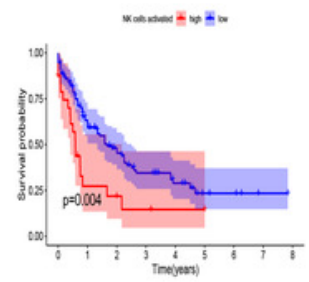

H
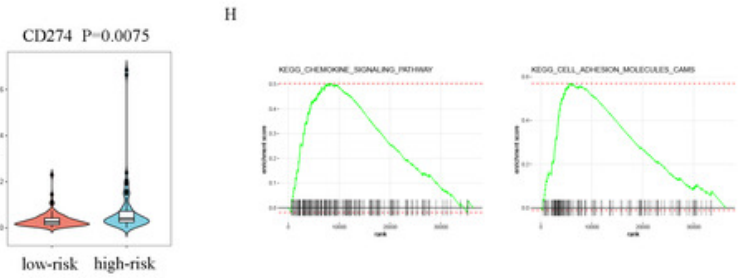
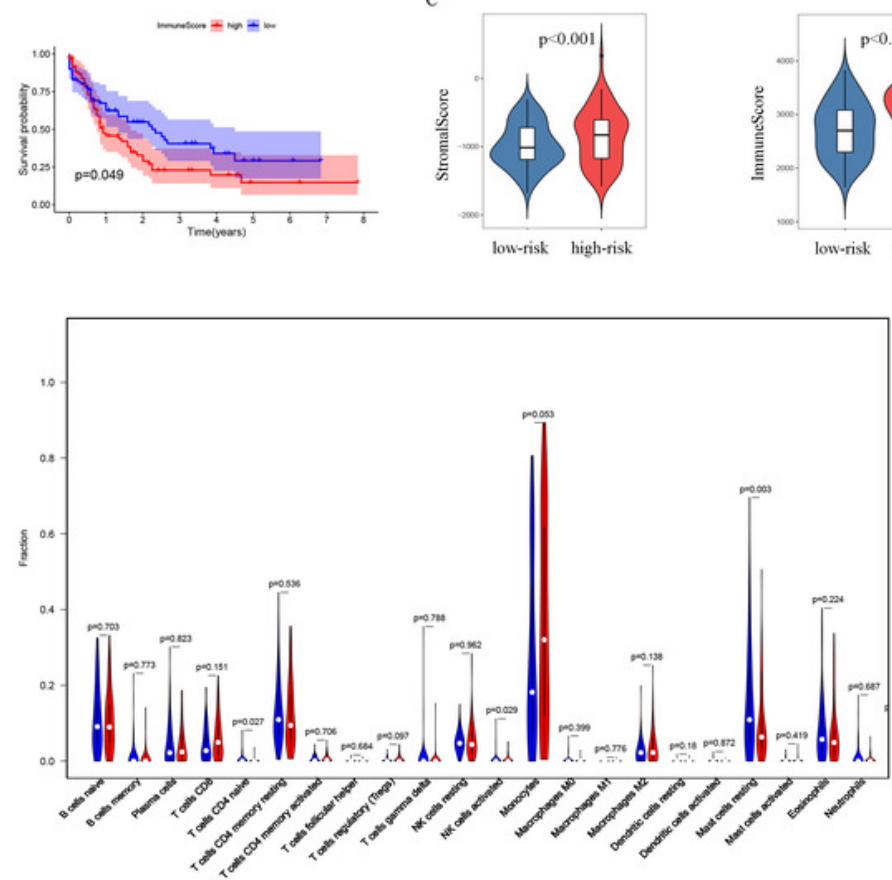

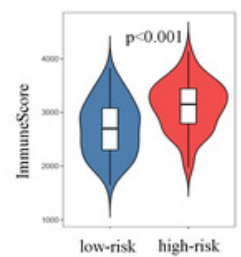

low-risk high-risk
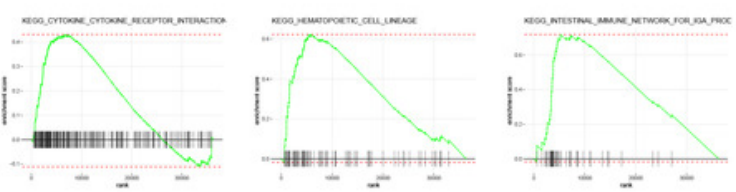
Figure 7

Drug sensitivity analysis to drugs of high- and low-risk subgroups.
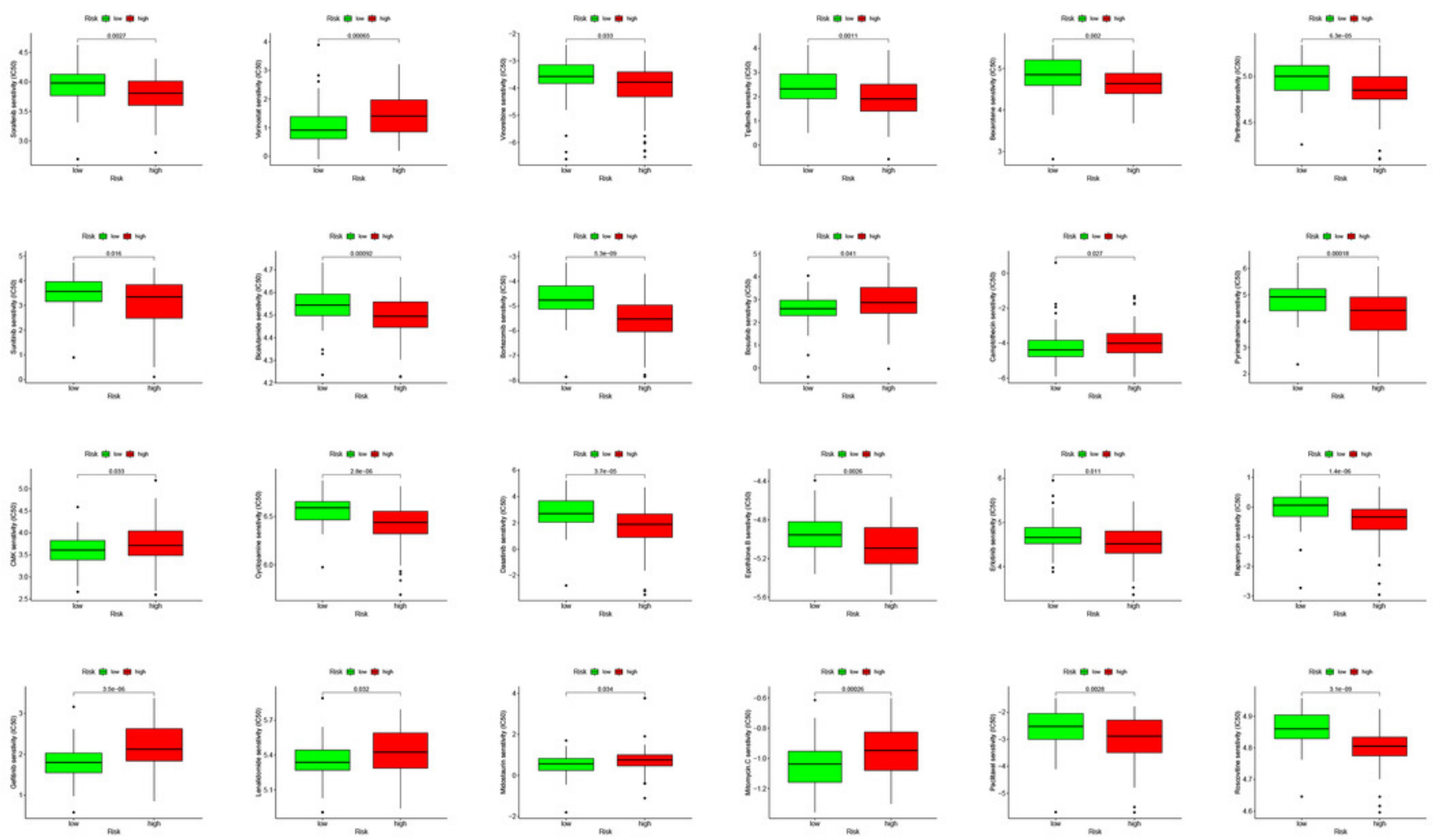